\title{
Creating an Emotionally Adaptive Game
}

\author{
Tim Tijs ${ }^{1}$, Dirk Brokken ${ }^{2}$, and Wijnand IJsselsteijn ${ }^{3}$ \\ ${ }^{1}$ User-System Interaction Program, Department of Industrial Design \\ Eindhoven University of Technology, P.O.Box 513, 5600MB, Eindhoven, The Netherlands \\ ${ }^{2}$ Philips Research Laboratories Europe \\ High Tech Campus 34, 5656 AE, Eindhoven, The Netherlands \\ ${ }^{3}$ Human-Technology Interaction Group, Department of Technology Management \\ Eindhoven University of Technology, P.O.Box 513, 5600MB, Eindhoven, The Netherlands \\ t.j.w.tijs@tue.nl, dirk.brokken@philips.com, \\ w.a.ijsselsteijn@tue.nl
}

\begin{abstract}
To optimize a player's experience, an emotionally adaptive game continuously adapts its mechanics to the player's emotional state, measured in terms of emotion-data. This paper presents the first of two studies that aim to realize an emotionally adaptive game. It investigates the relations between game mechanics, a player's emotional state and his/her emotion-data. In an experiment, one game mechanic (speed) was manipulated. Emotional state was self-reported in terms of valence, arousal and boredom-frustration-enjoyment. In addition, a number of (mainly physiology-based) emotion-data features were measured. Correlations were found between the valence/arousal reports and the emotion-data features. In addition, seven emotion-data features were found to distinguish between a boring, frustrating and enjoying game mode. Taken together, these features convey sufficient data to create a first version of an emotionally adaptive game.
\end{abstract}

Keywords: Adaptivity, personalization, computer games, affective loop, psychophysiology, emotions.

\section{Introduction}

For creating entertaining computer games ${ }^{1}$, gameplay is considered to be of key importance ([1], [2]). In absence of a broadly accepted definition of gameplay, we focus here on one frequently mentioned element of it, which is challenge. The process of optimizing a game's challenge is referred to as game balancing or difficulty scaling. That is, changing parameters in order to avoid that the player gets frustrated because the game is too hard, or gets bored because the game is too easy [3]. In this study, we have investigated the relations between a game's difficulty level and the interplay between the emotions boredom, frustration and enjoyment (Fig. 1-left panel). These

\footnotetext{
${ }^{1}$ From this point onward referred to as "games". In the present research, the term includes electronic games played on personal computers as well as those played on modern dedicated video game consoles, such as Sony's Playstation 3, Microsoft's Xbox 360 and Nintendo's Wii.
} 
relations strongly differ per individual, for example influenced by a player's skill level. For instance, a difficulty level that is found enjoyable by a novice might be boring for expert players. Games therefore need psychological customization techniques [4], such as difficulty adaptation, to optimize the experience. Since many years, game designers aim to provide some customization, for example by letting players choose a difficulty level upfront or including progressive difficulty levels during gameplay, based on a player's performance.

More advanced methods that work in real-time are less common. One difficulty adaptation mechanism, frequently applied in racing games, is called rubber banding [5]: When falling behind, the player suddenly gets an enormous boost in speed, which allows for catching up again (and vice versa for the competing cars). However, game adaptation that is solely based on in-game behavior can only have limited success, because there are many different types of players [6]. Each type of player has his/her own goals, preferences and emotional responses when playing a game. Hence, for optimizing the players' experiences, successful psychological customization requires a game to take the emotional state of the player into account. Games should become emotionally adaptive (Fig. 1- right panel).
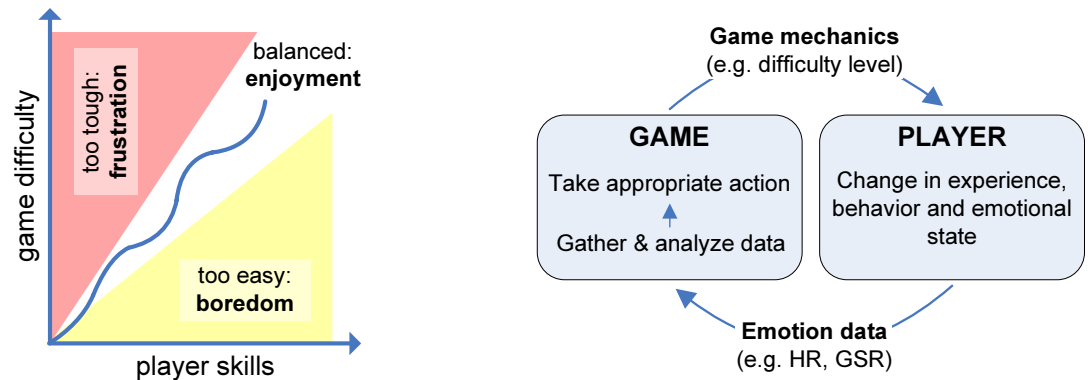

Fig. 1. Left panel: Game balancing (Adapted from [1]). Right panel: The emotionally adaptive games loop, inspired on the affective loop [7]

\subsection{Emotionally Adaptive Games}

The importance of emotions in computing is widely argued for (e.g. [8]). Emotion theorists differ over a discrete versus a dimensional model. The "discretionists" (e.g. [9]) argue for basic discrete emotions, such as anger, fear, sadness and happiness, as unique experiential states. The "dimensionalists" (e.g. [10]), on the other hand, look at emotions in terms of a two-dimensional space consisting of valence ("pleasantness") and arousal ("activation"). Sometimes dominance is added as a third dimension.

Effective human-computer interaction from an emotions perspective works in terms of an "affective loop" [7]. A similar feedback loop in a games context is described by [11]. Inspired by their work, Fig. 1 (right panel) shows a schematic view on the functioning of an emotionally adaptive game. By providing the right game mechanics [12] (e.g. audiovisuals, narrative, challenge), the game influences the player's experience, behavior and emotional state. Ideally, during play, the emotional state of the player (measured in terms of emotion-data), is continuously being fed back to the game so that the game can adapt its mechanics (e.g. difficulty level) 
accordingly in real-time. This all is done to create the optimal experience (which is referred to in literature as e.g. flow [13] or immersion [14]). Previous research attempts to create emotionally adaptive software have mainly focused on tutoring systems and workload / performance optimization (see e.g. [15]). Fewer attempts have been made to incorporate a closed-loop mechanism in a games context. Takahashi et al. [16] and Rani et al. [17] created a game that was found to improve player performance by adapting difficulty level to player's physiological state. Concept validation claims of these both studies were, however, based on a limited number of participants. Besides these attempts, a number of biofeedback games have recently been developed, which have some integration of a player's physiological data into the game (e.g. [18], [19] and [20]). These games however focus on stress manipulation rather than optimization of gameplay experience. Probably closest to the present project's scope is the work of Saari and colleagues, who created the Mind-Based Technology framework for psychological customization [21]. They have further elaborated this in a games context (e.g. [4], [22]) and are currently developing an emotionally adaptive game demo.

As a first step in creating an emotionally adaptive game, system input and output need to be specified in further detail. Regarding output (emotion-data), Saari et al. [22] provide an extensive discussion of possible elements to be adapted, structured by "psychologically validated templates". We have adopted a rather straightforward and intuitive "template": Game speed. We will manipulate the game's speed to influence the player's emotional state (the interplay between boredom, frustration and enjoyment, Fig. 1-left panel). Regarding system input (emotion-data), Öhman [23] distinguished three categories of emotion measures: Self-reports, overt behavior and physiological responses. Self-reports are frequently used for assessing players' emotions and experiences [5] but not suitable (since too obtrusive) for real-time application in a game. Regarding overt behavior, potentially useful techniques for measuring boredom, frustration and enjoyment are facial emotion tracking [24] and the analysis of posture and pressure exerted on the game controls [25]. Regarding physiological responses, there is an extensive field with many research findings in psychophysiology. Although the research is done in varying contexts with sometimes contradicting results, it is considered a highly interesting field for analyzing emotions in games. We have limited ourselves to the methods described below.

Regarding cardiovascular (heart) activity, tonic (long-term, as opposed to phasic) heart rate (HR) is known to increase with sympathetic nervous system activity, such as emotional arousal and cognitive effort and stress. On the other hand, increases in attention (mediated in the parasympathetic nervous system) lead to a decreased heart rate [26]. [27] found HR features to correlate with self-reported fun in games. Heart rate variability (HRV) is considered an index for mental effort (e.g. [28]). Some researchers (e.g. [29]) consider the percentage power in the low-frequency (LF) 0.07 $0.14 \mathrm{~Hz}$ range as a particularly effective index for task-related mental effort / sympathetic activity. Respiratory responses are analyzed to control for respiratory artifacts in e.g. HRV (a phenomenon known as respiratory sinus arrhythmia). Respiration may, however, also be used as a measure itself, e.g. for investigating stress and mental load [30]. Electrodermal activity (EDA) concerns the electrical resistance of the skin, also known as Skin Conductance (SCL, SCR) or Galvanic Skin Response (GSR). Skin conductance level is known to increase with information processing and the frequency 
of non-specific skin responses increases with arousal [26]. Electromyography (EMG) is a technique for measuring muscle activity; electric potential is being generated when muscle cells contract. Facial EMG is frequently used as a metric for valence. The most frequently analyzed facial muscles in this context are the orbicularis oculi (OO, used for closing the eyelids), zygomaticus major (ZYG, smiling) and corrugator supercilii (CORR, frowning). Most studies find positive correlations between valence and the $\mathrm{OO}$ and ZYG muscles, and a negative correlation between valence and CORR muscle (see e.g. [31], [32], [33]). In addition to the above findings, there are also a considerable number of studies without significant findings [34]. Because of the large differences in physiological responses between individuals and within individuals over time (autonomic response stereotypy principle, see e.g. [15], [35]), some researchers (e.g. [36]) argue for normalizing physiological data to facilitate a group analysis of the data. Additionally, affective systems should employ a battery of physiological features for accurate emotion predictions (e.g. [37]), and should allow for user-control for the sake of autonomy, privacy and interpretation of the data [41].

Because of the context-dependency of physiological responses, a two-stage approach was adopted. The purpose of the current initial study is to investigate physiological and other affect-related responses in relation to an experimentally induced change in game mechanics. Note that in this study the affective loop is not yet closed, that is, real-time affective indicators are not yet directly influencing the game mechanics. This will be the purpose of phase 2 of our work. The research question for the current invesigation evolved around the components of our framework (Fig. 1right panel): What game mechanics (speed settings) lead to what kind of emotional state, and what emotion-data is this accompanied by? This was investigated by means of a controlled experiment, as explained in the next section.

\section{Method}

\subsection{Participants}

In total 24 adults aged 23-46 (mean age 29.7) participated in the experiment; 19 males and 5 females. The participants played computer games (console, pc, arcade, and internet) for an average of 12.5 hours per month. All had played Pacman before. The participants were rewarded a €5 voucher for participation plus another €5 voucher for the final top-5 scores. All were informed in advance about this incentive. A withinsubjects design was applied using the game's speed-mode (3 levels: slow, fast and normal) as an independent variable. The order in which participants were confronted with the two non-normal speed modes (i.e. slow and fast) was counterbalanced over participants. A dual-PC setup was used to separate the game and questionnaire from the measurement software, to ensure the game and computer questionnaire to run smoothly.

\subsection{Design, Materials and Procedure}

The stimulus (game) was an adapted version of the PC-game Pacman [38]. Pacman is a relatively uncomplicated game without major changes in e.g. audiovisuals during 
play, which could lead to emotional bias. Moreover, it is a well-known game and is easy to pick up (requiring relatively short practice to minimize learning effects). Pacman also has a rather continuous flow of action (useful when comparing blocks of time) and has been used before in affective computing studies (e.g. [39]). To suit the game to the experiment, some adaptations were made: I) the player stayed in the same level during the experiment, II) objects that were eaten, such as points and pills, returned after a while), III) the speed level changed at preset times (unknown to the player), IV) eating objects increased the player's score, but being eaten meant a strong decrease in score, and V) the overall objective was to score as many points as possible. The choice for manipulating the difficulty parameter speed, instead of e.g. the number of opponents (i.e. ghosts) is based on the fact that the number of normal ghosts constantly changes during gameplay, because of Pacman eating star-shaped pills. Pacman was played using the arrow keys on the keyboard. All participants were instructed to play with their preferred hand.

The dependent variables comprised the player's emotional state and several emotion-data features. To gather demographics and gaming experience (i.e. gaming hours per month), a paper-questionnaire was filled out before the start of the game. Emotional state was measured through self-reporting in a computer-questionnaire (in between moments of play) and a post-game interview. The computer-questionnaire comprised two similar parts of three items, that focused on the minute of play before switching to this questionnaire. The first two items addressed valence and arousal, using Self-Assessment Manikins ([10], adapted to a five-point scale). The third was a multiple-choice item that a subject could use to indicate that (s)he was either enjoyed, bored or frustrated (or that none of these descriptions was applicable). The semistructured interview focused on the subject's overall opinion of the experiment and his or her preferred speed mode (fast, slow or normal). The emotion-data features that were collected during the game are displayed in Table 1.

Table 1. Emotion-data features analyzed in the present study

\begin{tabular}{lll}
\hline Measurement (sample freq in Hz) & Feature & Abbreviation \\
\hline Blood Volume Pulse (128) & BVP level & BVP \\
& BVP amplitude & BVP_AMP \\
& Heart rate & HR \\
& HRV-LF\% (0.04-0.15Hz) & HRV_LF \\
Respiration (32) & Respiration amplitude & RSP \\
& Respiration rate & RSP_RATE \\
& RSP-HR coherence & RSP_HR_COH \\
Skin Conductance (32) & Skin conductance level & SCL \\
& Number of SC responses & SCR \\
Facial Electromyography & Activity of the CORR & CORR \\
(1024) & Mean amplitude of the CORR & CORR_AMP \\
& Activity of the ZYG & ZYG \\
Keyboard Pressure (100) & Mean amplitude of the ZYG & ZYG_AMP \\
\hline
\end{tabular}


The physiological measurements were done using a wireless NeXus-10 kit. At the PC, the physiological data were stored using Biotrace+ v.1.16 . A BVP photoplethysmograph was placed on the ring finger of the subject's non-playing hand. A respiration belt was strapped around the thorax; a maximum of 2 layers of cloth was in between. Skin conductance sensors were placed on the tips of the index and middle finger of the non-playing hand. The Facial EMG electrodes ( $\mathrm{AgCl}, 15 \mathrm{~mm}$. diameter) were placed following instructions obtained from [40]. Keyboard pressure was measured by a pressure sensor placed under one of the keyboard's feet (the one closest to the arrow keys); keypresses were identified using a custom-made application. To synchronize the game events with the measurement data, a 3-window capture was implemented on the analysis PC.

Table 2 provides an overview of the test procedure.

Table 2. Test procedure

\begin{tabular}{lrr}
\hline Activity & min. \\
\hline Welcome, consent form, paper-questionnaire & $5-10$ \\
Installation of phys. equipm., game instructions, short practice & $5-10$ \\
Game part 1 & Normal speed & 2 \\
& Non-normal speed & 2 \\
Computer-questionnaire part 1 & Normal speed & 2 \\
Game part 2 & Non-normal speed & 2 \\
& & 2 \\
Computer-questionnaire part 2 & Normal speed & 1 \\
Game part 3 & 1 \\
Semi-structured post-game interview, debriefing & 5 \\
Total & & $\sim \mathbf{3 0}$ \\
\hline
\end{tabular}

\subsection{Analysis}

The feature analysis process consisted of a number of steps, as explained in Fig. 2.

\begin{tabular}{|c|c|c|c|c|c|c|c|c|c|c|}
\hline $\begin{array}{c}\text { Fragment } \\
\text { selection } \\
3 \times 60 \text { sec per } \\
\text { subject. }\end{array}$ & $\rightarrow$ & $\begin{array}{l}\text { Data filtering } \\
\text { e.g. to erase } \\
\text { movement } \\
\text { artifacts }\end{array}$ & $\rightarrow$ & $\begin{array}{c}\text { Outlier } \\
\text { inspection } \\
\text { performed } \\
\text { manually }\end{array}$ & $\rightarrow$ & $\begin{array}{c}\text { Normalization } \\
\text { for all features } \\
\text { except SCR }\end{array}$ & & $\begin{array}{l}\text { Calculation of } \\
\text { means } \\
\text { for all features } \\
\text { except SCR }\end{array}$ & $\rightarrow$ & $\begin{array}{c}\text { Statistical } \\
\text { analys is }\end{array}$ \\
\hline
\end{tabular}

Fig. 2. Analysis process

The three selected fragments per subject are from this point onward referred to as slow-mode, fast-mode and normal-mode. The physiological features were extracted and filtered using the default Biotrace+ detection and filtering algorithms. For the GSR, EMG and KEYB features, additional algorithms were used. The EMG signals (pre-amplified in the Nexus kit) were linearly rectified for analysis, high-pass filtered (Chebyshev Type 2, high-pass, cut-off frequency at $20 \mathrm{~Hz}$ ) and integrated (smoothened) using a $200 \mathrm{~ms}$ running average. For identifying skin conductance responses (SCR) and keypresses from the pressure recordings (KEYB), peak detection

\footnotetext{
${ }^{2}$ Both NeXus and Biotrace+ are manufactured by Mind Media b.v., The Netherlands.
} 
algorithms developed at Philips Research were used. Normalization of the physiological features, done according to [36], is required for a group analysis, because of the strong inter-subject differences in common physiological responses. These differences were also found for SCR; therefore SCR also requires a form of normalization. However, this could not be accomplished with the formula explained in [36], since one subject had in all three conditions (slow, fast, normal) the same number of identified skin conductance reponses. Therefore it was decided to normalize the SCR values for each subject over the subject's total signal span (3 conditions, thus 3 SCR values) in terms of inverted ranking ${ }^{3}$. Finally, statistical analysis was performed with SPSS v.16.0 software. As already mentioned in chapter 1, the analysis was focused on the relations between the various speed settings, the player's emotional state, and the accompanying emotion-data.

\section{Results}

Table 3 presents the self-report results from the questionnaire as well as from the post-game interview. Based on the results in Table 3, we draw the following conclusions: I) the participants felt bored in the slow-mode, II) in the fast-mode some were frustrated, others enjoyed themselves, and III) the normal-mode was preferred over the other two speed modes. Speculatively, we conclude the following: the slow-mode was too slow, the fast-mode was for some a bit too fast, but for others the right speed level. The speed level in the normal-mode might not be optimal either, but the players' experiences are better in that mode than in the other two.

Table 3. Self-report results

\begin{tabular}{|c|c|c|c|c|}
\hline \multicolumn{5}{|c|}{ Computer-questionnaire ("I felt..." - count) } \\
\hline & bored & frustrated & enjoyed & not applicable \\
\hline slow-mode & 20 & 2 & 2 & 0 \\
\hline fast-mode & 2 & 8 & 14 & 0 \\
\hline \multicolumn{5}{|c|}{ Post-game interview (preferred speed mode - count) } \\
\hline \multicolumn{5}{|c|}{$\begin{array}{l}\text { Post-game interview (preferred speed mode - count) } \\
\text { slow-mode }\end{array}$} \\
\hline 0 & \multicolumn{2}{|l|}{1} & \multicolumn{2}{|l|}{23} \\
\hline
\end{tabular}

There was a tendency toward neutral scoring on the SAM (valence and arousal) topics. For instance, the 22 non-enjoyment scores (i.e. reports of boredom or frustration) in the slow-mode were accompanied by only 5 negative valence reports. To investigate the relations between the SAM (valence and arousal) scores and the emotion-data features, both valence scores and both arousal scores for a subject were averaged. A Pearson correlation test revealed two statistically significant results $(\mathrm{p}<0.050)$, as displayed in Fig. 3. Firstly, arousal correlated with the emotion-data feature BVP $(r=0.449, p=0.032, \mathrm{~N}=23)$. Since the arousal score of 1 represents the

\footnotetext{
${ }^{3}$ I.e.: the highest of the 3 SCR values for a subject was awarded the rank 3 and the lowest score of the three scores the rank 1. For instance, the SCR values 3 (slow-mode) ,4 (fast-mode) and 1 (normal-mode) for a subject were respectively transformed into the normalized values 2,3 and 1. Similarly, a subject's SCR values of 10, 10 and 10 were transformed into 2, 2 and 2.
} 
highest arousal report, this correlation should be interpreted as "the higher the arousal report, the higher the BVP value of a player in the non-normal game modes". Secondly, valence appeared to correlate negatively with the ZYG_AMP feature $(\mathrm{r}=-0,446, \mathrm{p}=0,033, \mathrm{~N}=23)$. This result should be read as "the more positive valence reports in the non-normal speed modes were accompanied by larger movements in the zygomaticus major muscle". When observing this correlation in Fig. 3 (right panel), it seems that two data points (squares in both lower corners) have a strong influence on the correlation effect. Although these data points lay rather far out, they were kept in the analysis because I) both represent an average of two conditions, II) the video recordings of the related participants do not reveal any anomalies and III) strong inter-subject differences in physiological responses are common (e.g. [15]).
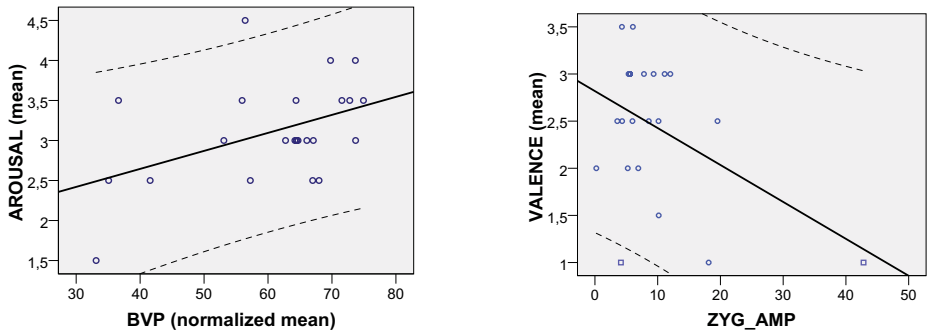

Fig. 3. Significant correlations between the SAM scales and emotion-data features. For the valence and arousal axes: $1=$ Most positive/highest, $5=$ least positive/lowest. Dotted lines represent confidence intervals $(95 \%)$.

Table 4. Emotion-data features that distinguish between the game's speed modes

\begin{tabular}{|c|c|c|c|c|c|}
\hline Feature & Conditions & $\mathbf{t}$ & df & $\mathbf{p}$ & mode identified (desired action) \\
\hline \multirow[t]{2}{*}{ SCL } & slow-fast & -2.511 & 22 & 0.020 & slow (speed up) \\
\hline & slow-normal & -4.197 & 22 & 0.000 & \\
\hline \multirow[t]{2}{*}{ SCR } & slow-fast & -4.851 & 22 & 0.000 & slow (speed up) \\
\hline & slow-normal & -4.343 & 22 & 0.000 & \\
\hline \multirow[t]{2}{*}{ HR } & slow-fast & -5.177 & 22 & 0.000 & slow (speed up) \\
\hline & slow-normal & -5.196 & 22 & 0.000 & \\
\hline \multirow[t]{2}{*}{ RSP_RATE } & slow-fast & -3.806 & 22 & 0.001 & slow (speed up) \\
\hline & slow-normal & -2.388 & 22 & 0.026 & \\
\hline \multirow[t]{2}{*}{ CORR_AMP } & slow-fast & 3.078 & 22 & 0.006 & fast (slow down) \\
\hline & fast-normal & -3.592 & 22 & 0.002 & \\
\hline \multirow[t]{2}{*}{ ZYG_AMP } & slow-fast & -2.673 & 22 & 0.014 & fast (slow down) \\
\hline & fast-normal & 2.767 & 22 & 0.011 & \\
\hline \multirow[t]{3}{*}{ KEYB } & slow-fast & -5.136 & 22 & 0.000 & slow (speed up) \& fast (slow \\
\hline & slow-normal & -3.637 & 22 & 0.001 & down) \& normal (none) \\
\hline & fast-normal & 3.282 & 22 & 0.003 & \\
\hline
\end{tabular}

To analyze the effects of speed on the players' emotion-data, a repeated measures ANOVA was performed with speed (3 levels: slow, fast, normal) as a within subjects factor. This provided an overview with a number of significant effects. To analyze these effects (i.e. the differences in emotion-data between the 3 speed modes) in more 
detail, a series of paired sample t-tests was performed. Significant results $(\mathrm{p}<0.050)$ are provided in Table 4. For example, Table 4 and Fig. 4 show that the average skin conductance level (SCL) during the slow-mode was significantly lower than during the other two speed modes. Since the participants reported boredom during the slowmode, our conclusion is that this mode was too slow. In other words, the game should speed up. According to Table 4, keyboard pressure (KEYB) can even help to distinguish between the game's 3 speed levels.
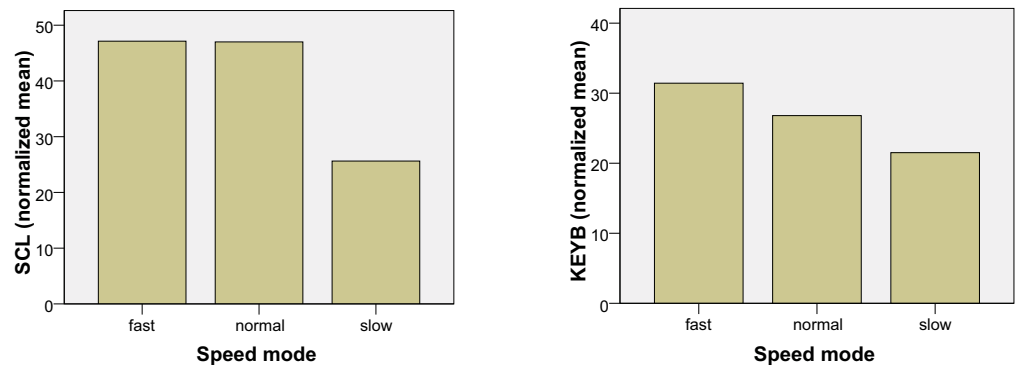

Fig. 4. Significantly different emotion-data SCL (left panel) and KEYB (right panel)

\section{Discussion and Further Research}

When comparing our findings to other studies that investigate in the relations between valence, arousal and physiological responses, one result seems to contradict most other work: The positive correlation between self-reported arousal and BVP (Fig. 3left panel). In addition to the central tendency in scoring on valence and arousal, no less than 8 of the 24 participants asked for the exact meaning of the arousal manikins when first confronted with them. Two even thought that the highest-arousal manikin was supposed to depict hunger. In turn, the experimenter described it to them as their level of activation.

The induction of boredom, frustration and enjoyment by manipulation of the game mechanic "speed" was partly successful. Nearly all players indicated to be bored during the slow-mode, but the fast-mode was found more enjoyable than frustrating. There are several possible causes for this. Firstly, the players knew the game speed was going to change. Besides, the speed changes were rather abrupt, and the players knew it only lasted for a limited amount of time. Nonetheless, nearly all participants (see Table 3) considered the normal-mode the most enjoyable of the three speed modes.

As a stimulus, Pacman appeared to be a successful game choice. No major problems (such as crashing software or a stalemate situation in the game) were encountered during the experiment. Besides, from the post-game interviews, we conclude that all participants enjoyed the game from an overall perspective. It was also relatively easy to implement the speed manipulations and customized ruleset in the game.

In the next phase of this project, we will aim to develop an emotionally adaptive version of the Pacman game. This game will use the data collected in the present study as a starting (or: calibration) point for adaptation. For instance, when the 
emotionally adaptive Pacman game receives emotion-data that matches the data pattern found for the "boring" slow-mode (e.g. relatively low SCL and SCR values, see Table 4), it will either increase its speed or, to preserve player autonomy [41], subtly ask the user whether it should increase its speed.

However, such a classification system will only work properly when it has sufficient input data (i.e. sufficient emotion-data features that are known to distinguish between the states of enjoyment, frustration and boredom) from a sufficient amount of participants. Therefore, the present dataset will be analyzed in more detail, to find more useful features. This will e.g. include the analysis of the video recordings (through facial emotion tracking) and the analysis of in-game data that were gathered during the experiment. The measurements described in Table 1 will also be explored in more detail, by e.g. analyzing standard deviation, variance, kurtosis and skewness of the data (see e.g. [42], [43]). In addition to the present physiological state, another possibly useful source of input data lies in the initial or previous emotional states (e.g. [44], [45]). For example, a high (e.g. 140 BPM) heart rate might not increase as much by an arousing game effect as a normal heart rate. Besides more emotion-data features from more participants, also different data features are required. In the present experiment, the participants could only report on their gameplay experiences during two rather extreme speed modes (very slow and very fast). Since an emotionally adaptive game should undertake action as soon as the enjoyment borders (Fig. 1-left panel) are crossed, accurate emotion-data on these crossing-border events will be needed.

\section{Acknowledgments}

The authors would like to thank Gert-Jan de Vries, Jack van den Eerenbeemd, Paul Lemmens and Floris Crompvoets of Philips Research Laboratories for their contributions to the project.

\section{References}

1. Adams, E., Rollings, A.: Game Design and Development; Fundamentals of Game Design. Pearson Education, NJ (2007)

2. Malone, T.W.: What Makes Computer Games Fun? Byte 6, 258-277 (1981)

3. Koster, R.: Theory of Fun for Game Design. Paraglyph Press, Phoenix (2004)

4. Saari, T., Ravaja, N., Laarni, J., Kallinen, K., Turpeinen, M.: Towards Emotionally Adapted Games. In: Proceedings of Presence 2004, Valencia, Spain, pp. 182-189 (2004)

5. Pagulayan, R.J., Keeker, K., Wixon, D., Romero, R., Fuller, T.: User-centered design in games. In: Jacko, J., Sears, A. (eds.) Handbook for Human-Computer Interaction in Interactive Systems, pp. 883-906. Lawrence Erlbaum Associates Inc., Mahwah (2002)

6. Bartle, R.A.: Hearts, Clubs, Diamonds, Shades: Players who suit MUDs, http: / /www.mud.co.uk/richard/hcds.htm

7. Sundström, P., Ståhl, A., Höök, K.: eMoto - A User-Centred Approach to Affective Interaction. In: Tao, J., Tan, T., Picard, R.W. (eds.) ACII 2005. LNCS, vol. 3784. Springer, Heidelberg (2005)

8. Picard, R.W.: Affective Computing. MIT Press, Cambridge (1997)

9. Ekman, P.: An Argument for Basic Emotions. Cognition and Emotion 6, 169-200 (1992) 
10. Lang, P.J.: The emotion probe: Studies of Motivation and Attention. American Psychologist 50, 372-385 (1995)

11. Polaine, A.: The flow principle in interactivity. In: Proceedings of 2nd Australasian Conference on Interactive Entertainment, Sydney, Australia, pp. 151-158 (2005)

12. Hunicke, R., LeBlanc, M., Zubek, R.: MDA: A Formal Approach to Game Design and Game Research. In: Proceedings of the Challenges in Game AI Workshop. Nineteenth National Conference on Artificial Intelligence, San Jose, CA (2004)

13. Csikszentmihalyi, M.: Finding flow: The Psychology of Engagement with Everyday Life. Basic Books, NY (1997)

14. Ermi, L., Mäyrä, F.: Proceedings of DiGRA 2005, Vancouver, Canada (2005)

15. Schaefer, F., Haarmann, Boucsein, W.: The Usability of Cardiovascular and Electrodermal Measures for Adaptive Automation. In: Westerink, J.H.D.M., Ouwerkerk, M., Overbeek, T.J.M., Pasveer, W.F., De Ruyter, B. (eds.) Probing Experience: From Assessment of User Emotions and Behavior to Development of Products. Philips Research Book Series, vol. 8, pp. 235-243 (2008)

16. Takahashi, M., Tsuyoshi, A., Kuba, O., Yoshikawa, H.: Experimental Study Toward Mutual Adaptive Interface. In: Proceedings of the 3rd IEEE International Conference on Robot and Human Communication, Nagoya, Japan, pp. 271-276 (1994)

17. Rani, P., Sarkar, N., Liu, C.: Maintaining Optimal Challenge in Computer Games Through Real-Time Physiological Feedback. In: Proceedings of the 1st International Conference on Augmented Cognition, Las Vegas, NV (2005)

18. Journey to Wild Divine, http: / / www . wilddivine.com

19. Bersak, D., McDarby, G., Augenblick, N., McDarby, P., McDonnell, D., McDonald, B., Karkun, R.: Intelligent Biofeedback Using an Immersive Competitive Environment. In: Abowd, G.D., Brumitt, B., Shafer, S. (eds.) UbiComp 2001. LNCS, vol. 2201. Springer, Heidelberg (2001)

20. BioTetris, http://www.ncsx.com/www/ncs110998/tetris_64.htm

21. Saari, T.: Mind-Based Media and Communications Technologies. How the Form of Information Influences Felt Meaning. Acta Universitatis Tamperensis 834. Tampere University Press, Tampere (2001)

22. Saari, T., Ravaja, N., Turpeinen, M., Kallinen, K.: Emotional Regulation System for Emotionally Adapted Games. In: Proceedings of FuturePlay 2005, Michigan State University, MI (2005)

23. Öhman, A.: The Psychophysiology of Emotion: An Evolutionary-Cognitive Perspective. In: Ackles, P.K., Jennings, J.R., Coles, M.G.H. (eds.) Advances in Psychophysiology, vol. 2, pp. 79-127. JAI Press, Greenwich (1987)

24. Fasel, B., Luettin, J.: Automatic Facial Analysis: A Survey. IDIAP Research Report (IDIAP RR 99-19). Pattern Recognition 36(1), 259-275 (2003)

25. Van den Hoogen, W., IJsselsteijn, W.A., de Kort, Y.A.W., Poels, K.: Towards Real-Time Behavioral Indicators of Player experiences: Pressure patterns and Postural Responses. In: Proceedings of the 6th International Conference on Methods and Techniques in Behavioral Research, Maastricht, The Netherlands (2008)

26. Ravaja, N.: Contributions of Psychophysiology to Media Research: Review and Recommendations. Media Psychology 6, 193-235 (2004)

27. Yannakakis, G.N., Hallam, J., Lund, H.H.: Entertainment Capture through Heart Rate Activity in Physical Interactive Playgrounds. User Modeling and User-Adapted Interaction, Special Issue on Affective Modeling and Adaptation 18(1-2), 207-243 (2008)

28. Vicente, K.J., Thornton, D.C., Moray, N.: Spectral Analysis of Sinus Arrhythmia: A Measure of Mental Effort. Human Factors 29(2), 171-182 (1987) 
29. Veltman, J.A., Gaillard, A.W.K.: Indices of Mental Workload in a Complex Task Environment. Neuropsychobiology 28, 72-75 (1993)

30. Wientjes, C.J.E.: Respiration in Psychology: Methods and Applications. Biological Psychology 34, 179-204

31. Mahlke, S., Minge, M., Thüring, M.: Measuring Multiple Components of Emotions in Interactive Contexts. In: Extended Abstracts of CHI 2006, Montréal, Canada (2006)

32. Partala, T.: Affective Information in Human-Computer Interaction. Academic Dissertation, Department of Computer Sciences, University of Tampere, Finland (2005)

33. Hazlett, R.L., Benedek, J.: Measuring emotional valence to understand the user's experience of software. Int. J. Hum.-Comput. Stud. 65(4), 306-314 (2007)

34. Luczak, H., Göbel, M.: Signal Processing and Analysis in Application. In: Backs, R.W., Boucsein, W. (eds.) Engineering Psychophysiology: Issues and Applications, pp. 79-110. Lawrence Erlbaum, Mahwah (2000)

35. Lacey, J.I., Lacey, B.C.: Verification and extension of the principle of autonomic response-stereotypy. Am. J. Psychol. 71, 50-73 (1958)

36. Mandryke, R.L., Atkins, M.S.: A Fuzzy Physiological Approach for Continuously Modeling Emotion During Interaction with Play Technologies. International Journal of HumanComputer Studies 65, 329-347 (2007)

37. Thayer, J.F., Friedman, B.H.: The Design and Analysis of Experiments in Engineering Psychophysiology. In: Backs, R.W., Boucsein, W. (eds.) Engineering Psychophysiology: Issues and Applications, pp. 59-78. Lawrence Erlbaum, Mahwah (2000)

38. Overmars, M., McQuown, B.: Pacman.GM6 [computer software]

39. Yannakakis, G.N., Hallam, J.: Towards Optimizing Entertainment in Computer Games. Applied Artificial Intelligence 21, 933-971 (2007)

40. Waterink, W.: Facial Muscle Activity as an Index of Energy Mobilization During Processing of Information: An EMG Study. Doctoral Thesis, Tilburg University, Tilburg, The Netherlands (1997)

41. Höök, K., Ståhl, A., Sundström, P., Laaksolahti, J.: Interactional Empowerment. In: Proceedings of CHI 2008, Florence, Italy, pp. 647-656. ACM Press, New York (2008)

42. Picard, R., Vyzas, E., Healey, J.: Toward Machine Emotional Intelligence: Analysis of Affective Physiological State. IEEE Transactions on Pattern Analysis and Machine Intelligence 23(10), 1175-1191 (2001)

43. Van den Broek, E.L., Schut, M.H., Westerink, J.H.D.M., Van Herk, J., Tuinenbreijer, K.: Computing Emotions Awareness through Facial Electromyography. In: Huang, T.S., Sebe, N., Lew, M., Pavlović, V., Kölsch, M., Galata, A., Kisačanin, B. (eds.) ECCV 2006 Workshop on HCI. LNCS, vol. 3979(12), pp. 52-63. Springer, Heidelberg (2006)

44. Wilder, J.: Stimulus and Response: The Law of the Initial Value. J Wright, Bristol, England (1967)

45. Berntson, G.G., Cacioppo, J.T., Quigley, K.S.: Autonomic Determinism: The Modes of Autonomic Control, the Doctrine of Autonomic Space, and the Laws of Autonomic Constraint. Psychological Review 98(4), 459-487 (1991) 